\title{
On the Link Space of a Gorenstein Quasihomogeneous Surface Singularity
}

\author{
Igor V. Dolgachev * \\ Department of Mathematics, University of Michigan, Ann Arbor, MI 48109, USA
}

\section{Introduction}

Let $(X, o) \subset\left(\mathbb{C}^{n}, 0\right)$ be a normal quasihomogeneous surface singularity and $M$ be its link space. Recall that $M$ is a compact smooth 3-manifold obtained by interesting $X$ with a small $(2 n-1)$-sphere centered at the origin or by dividing $X^{*}=X-\{0\}$ by the action of the subgroup $\mathbb{R}_{+}$of $\mathbb{C}^{*}$. It is known [8] that the analytic structure on $X^{*}$ defines a geometric structure on $M$, i.e. a complete locally homogeneous riemannian metric of finite volume on $M$. In particular, the universal cover of $M$ is a homogeneous space. It was shown by Milnor [4] that in the special case, where $(X, o)$ is given by an equation $z_{1}^{p}+z_{2}^{q}+z_{3}^{r}=0$ the link space $M$ is homogeneous itself and diffeomorphic to a coset space $\Gamma \backslash G$. Here $G$ is a simply connected 3-dimensional Lie group and $\Gamma$ its discrete cocompact subgroup. In this paper we prove the following generalization of this result :

Theorem. Let $(X, o)$ be a Gorenstein quasihomogeneous surface singularity and $M$ be its link space. Then $M$ is diffeomorphic to a coset space $\Gamma \backslash G$, where $G$ is a simply connected 3-dimensional Lie group and $\Gamma$ its discrete cocompact subgroup. Conversely, if $M$ is the link space of a normal quasihomogeneous surface singularity and $M$ is diffeomorphic to a coset space as above, then $M$ is the link space of a Gorenstein quasihomogeneous surface singularity.

Recall that a normal surface singularity $(X, o)$ is called a Gorenstein singularity if there exists a holomorphic 2-form on $X^{*}$ which does not vanish anywhere. Every complete intersection singularity, in particular, a hypersurface singularity, is a Gorenstein singularity.

As was pointed out to me by F. Ehlers and J. Scherk, the diffeomorphism between the link space $M$ of a Gorenstein quasihomogeneous singularity and the space $\Gamma \backslash G$ not only preserves the geometric structures but also the $C R$-structures. The $C R$-structure on $M$ is defined by the analytic structure on $X^{*}$ and determines the singularity $(X, o)$ up to an analytic isomorphism [15]. The $C R$-structure on

* Research partially supported by NSF grant MCS 80-02272 
$\Gamma \backslash G$ is defined by the unique $G$-invariant $C R$-structure on $G$ [1]. It would be very interesting to find out an intrinsic characterization of the $C R$-structure on the link space of a Gorenstein singularity.

The pairs $(G, \Gamma)$ which occur in the theorem are of the following three types:

I) $G=\mathrm{SU}(2), \Gamma$ any finite subgroup;

II) $G=\operatorname{PSL}(2, \mathbb{R})^{\sim}$, the universal cover of $\operatorname{PSL}(2, \mathbb{R}), \Gamma$ is the corresponding extension of a fuchsian subgroup $\bar{\Gamma}$ of $\operatorname{PSL}(2, \mathbb{R})$ of signature $\left(g ; e_{1}, \ldots, e_{r}\right)$;

III) $G$ is the Heisenberg group, the group of matrices

$$
[a, b, c]=\left(\begin{array}{ccc}
1 & a & c \\
0 & 1 & b \\
0 & 0 & 1
\end{array}\right), \quad a, b, c \in \mathbb{R},
$$

$\Gamma$ is the subgroup of matrices $[a, b, c]$ with $a, b, c \in k \mathbb{Z}$ for some integer $k$.

We will call a pair $(G, \Gamma)$ as above an admissible pair. In the Cases I) and II), $g=0$, the pair $(G,[\Gamma, \Gamma])$ is admissible if $(G, \Gamma)$ is admissible. It corresponds to a complete intersection singularity of the form:

$$
z_{1}^{e_{1}}+z_{i}^{e_{i}}+a_{i} z_{r}^{e_{r}}=0, i=2, \ldots, r-1, a_{i} \in \mathbb{C}^{*} .
$$

This was proven by Milnor in the case $r=3$ [4] and generalized to the case $r>3$ by Neumann [6]. One can find the above equations and a proof of a closely related result (via Sect. 2 of this paper) in the work of Poincaré [11] (cf. [3]).

Apart from Gorenstein quasihomogeneous singularities there is another class of surface singularities whose link space is diffeomorphic to a coset space $\Gamma \backslash G$, where $G$ is a 3-dimensional simply connected Lie group and $\Gamma$ its cocompact discrete subgroup. This is the class of cusp singularities (which are always Gorenstein singularities). We discuss this case in Sect. 3 of the paper.

The main result of this paper may be viewed as an algebraic geometrical version of a result of Raymond and Vasquez [12]. At the same time and independently it was also obtained by W. Neumann (unpublished). I am thankful to C. T. C. Wall for awaking my interest to these questions by showing his proof of the main result of this paper in the case of complete intersection singularities.

\section{Gorenstein Automorphy Factors}

In this section we recall the construction of a uniformization of normal quasihomogeneous surface singularities from $[2,10]$ and prove a result (Proposition 1) first announced in [3].

Let $\boldsymbol{P}$ be one of the three standard simply connected complex manifolds of dimension $1: \mathbb{P}^{1}(\mathbb{C}), H=\{z \in \mathbb{C}: \operatorname{Im}(z)>0\}, \mathbb{C}$. Let $\bar{\Gamma}$ be a discrete subgroup of $\operatorname{Aut}(P)$ with the compact quotient $C=P / \bar{\Gamma}$ and $L$ be a non-ramified automorphy factor on $P$ with respect to $\bar{\Gamma}$. The latter means that $L$ is a line $\bar{\Gamma}$-bundle on $P$ and the action of $\vec{\Gamma}$ on $L^{*}=L-$ zero section $\}$ is fixed-point-free (this condition was meant but omitted in [2]). It is well-known that $\bar{\Gamma}$ contains a normal subgroup of finite index $\bar{\Gamma}^{\prime}$ which acts freely on $P$. Let $L^{\prime}=L / \bar{\Gamma}^{\prime}$ be the non-ramified automorphy factor on $C^{\prime}=C / \bar{\Gamma}^{\prime}$ with respect to the group $G=\bar{\Gamma} / \bar{\Gamma}^{\prime}$. We call the pair $\left(L^{\prime}, G\right)$ obtained in this way the standard reduction of the pair $(L, \bar{\Gamma})$. Assume 
now that $L^{\prime}$ is a negative line bundle on $C^{\prime}$ (i.e. $L^{\prime-1}$ is an ample line bundle). This condition is independent of a choice of the standard reduction, we call $L$ a negative automorphy factor if it is satisfied. Then the graded algebra $A(\bar{\Gamma} ; L)$ $=\bigoplus_{m=0}^{\infty} H^{0}\left(P, L^{-m}\right)^{\bar{\Gamma}}=\bigoplus_{m=0}^{\infty} H^{0}\left(C^{\prime}, L^{\prime-m}\right)^{G}$ is a normal finitely generated graded $\mathbb{C}$-algebra. Its affine spectrum $X(\bar{\Gamma} ; L)$ is an affine surface with an isolated singularity at the point $o \in X(\bar{\Gamma} ; L)$ corresponding to the maximal ideal $A(\bar{\Gamma} ; L)_{>0}$ $=\bigoplus_{m=0}^{\infty} A(\bar{\Gamma} ; L)_{m>0}$. It follows from the definition that

$$
X(\bar{\Gamma} ; L)^{*}:=X(\bar{\Gamma} ; L)-\{o\} \simeq L^{*} / \bar{\Gamma} .
$$

In particular, the universal cover of $X(\bar{\Gamma} ; L)^{*}$ coincides with the universal cover of $L^{*}$ and the fundamental group $\pi_{1}\left(X(\bar{\Gamma} ; L)^{*}\right)$ is an extension of $\bar{\Gamma}$ with help of the cyclic group $K=\pi_{1}\left(L^{*}\right)$. The singularity $(X(\bar{\Gamma} ; L), o)$ is a quasihomogeneous singularity, the corresponding good $\mathbb{C}^{*}$-action is defined by the grading of the ring $A(\bar{\Gamma} ; L)$. Its link space $M$ is diffeomorphic to the quotient space $X(\bar{\Gamma} ; L)^{*} / \mathbb{R}_{+}$. This implies that

$$
M \underset{\text { diff }}{\approx}\left(L^{*} / \mathbb{R}_{+}\right)^{\sim} / \Gamma
$$

where $\mathbb{R}_{+}$acts on $L^{*}$ via the canonical $\mathbb{C}^{*}$-action on $L^{*}$, stands for the universal cover, and $\Gamma=\pi_{1}\left(X(\bar{\Gamma} ; L)^{*}\right)$.

Now, let $(X, o)$ be an arbitrary normal quasihomogeneous surface singularity. According to $[2,10]$ there exists a unique non-ramified negative automorphy factor $L$ on $P$ and a discrete group $\bar{\Gamma}$ of automorphisms of $P$ such that

$$
(X, o) \simeq(X(\bar{\Gamma} ; L), o) .
$$

This gives a description of the diffeomorphism type of the link space $M$ of $(X, o)$. In the case, where $(X, o)$ is Gorenstein we will identify the space $L^{*} / \mathbb{R}_{+}$with a 3-dimensional Lie group $G$, the group $\bar{\Gamma}$ with its discrete subgroup, and the action of $\bar{\Gamma}$ with the left-multiplication action. First, we need the following simple lemma :

Lemma 1. Let $L^{\prime}$ be a negative line bundle on a complete algebraic curve $C^{\prime}$ and $(X, o)$ be the normal singularity obtained by blowing down the zero section of $L^{\prime}$ (i.e. $X$ is the affine spectrum of the ring $\left.\bigoplus_{m=0}^{\infty} H^{\circ}\left(C^{\prime}, L^{\prime-m}\right)\right)$. Then $(X, o)$ is a Gorenstein singularity if and only if some tensor power $L^{\prime m}$ of $L^{\prime}$ is isomorphic to the tangent bundle $T_{C^{\prime}}$ of $C^{\prime}$.

Proof. Let $U=L^{\prime *}=L^{\prime}-$ zero section $\}$ and $q: U \rightarrow C^{\prime}$ be the canonical projection. The natural $\mathbb{C}^{*}$-action on $U$ trivializes the relative cotangent bundle $T_{U / C^{\prime}}$. This immediately implies that $\bigwedge_{\wedge}^{2} T_{U}=q^{*}\left(T_{C^{\prime}}\right)$. Passing to the associated dual sheaves, we get

$$
w_{U}=q^{*}\left(w_{C}\right)
$$


Taking the direct images of the both sides, we obtain

$$
q_{*}\left(\omega_{U}\right)=q_{*}\left(q^{*}\left(\omega_{C^{\prime}}\right)\right)=\omega_{C^{\prime}} \otimes q_{*}\left(\omega_{V}\right)=w_{C^{\prime}} \otimes\left(\bigoplus_{m \in \mathbb{Z}} L^{m}\right)=\bigoplus_{m \in \mathbf{Z}}\left(w_{C^{\prime}} \otimes L^{m}\right) .
$$

Assume that $(X, o)$ is Gorenstein. Then $w_{V} \simeq \mathscr{O}_{U}$ and

$$
q_{*}\left(\omega_{U}\right)=\bigoplus_{m \in \mathbf{Z}} L^{m} \simeq \bigoplus_{m \in \mathbf{Z}}\left(w_{C} \otimes L^{m}\right) .
$$

It obviously implies that $\omega^{\prime} C^{\prime} \otimes L^{m} \simeq \mathscr{O}_{C^{\prime}}$ for some integer $m$, i.e. $L^{m} \simeq T_{C^{\prime}}$. Conversely, if the latter is true, then

$$
\bigwedge^{2} T_{U}=q^{*}\left(T_{C^{\prime}}\right) \simeq q^{*}\left(L^{m}\right)=1_{U},
$$

the trivial bundle, and $(X, o)$ is Gorenstein.

Lemma 2. In the notation of Lemma 1, assume that $L^{\prime}$ has a structure of an automorphy factor with respect to a finite group $G$ of automorphisms of $C^{\prime}$. Then the conclusion of Lemma 1, where the isomorphism means a G-equivariant isomorphism, is still valid.

Proof. We leave it to the reader who can easily verify that all the isomorphisms in the proof of Lemma 1 can be made $G$-equivariant.

Proposition 1. Let $(X(\bar{\Gamma} ; L), o)$ be the normal quasihomogeneous surface singularity associated to a negative non-ramified automorphy factor on $P$ and a discrete subgroup $\bar{\Gamma}$ of $\operatorname{Aut}(P)$. Then $(X(\bar{\Gamma} ; L), o)$ is a Gorenstein singularity if and only if $L^{m} \simeq T_{p}$ (as $\bar{\Gamma}$-bunldes) for some $m \in \mathbb{Z}$ and $\bar{\Gamma}$ is contained in the translation subgroup of $\operatorname{Aut}(P)$ in the case $P=\mathbb{C}$ (notice that in the latter case $L^{0}=1_{P} \simeq T_{P}$ ).

Proof. Let $P=\mathbb{P}^{1}(\mathbb{C})$. In this case the assertion is equivalent to the well-known result of Watanabe. To be self-contained, let us prove it. Since $\operatorname{deg}\left(T_{P}\right)=2$ and $L$ is negative, we must have $m=-1$ or -2 . Suppose that $m=-1$, i.e. $L \simeq T_{P}^{-1}$, the cotangent bundle of $P$. Let $\left(z, t_{z}\right)$ be local coordinates on $L$. Then the group $\bar{\Gamma}$ acts on $L$ by sending $(z, t)$ to $\left(g(z), \dot{g}(z) t_{g(z)}\right), g \in \bar{\Gamma}$ (we identify $t_{z}$ with $\left.\frac{\delta}{\delta z}\right)$. This implies that the 2-form $d z \wedge d t_{z}$ is defined globally on $T_{P}^{-1}$ and is invariant with respect to $\bar{\Gamma}$ (it defines a structure of a symplectic manifold on $T_{P}^{-1}$ with respect to which $\bar{\Gamma}$ acts on $T_{P}^{-1}$ by symplectic automorphisms). This 2-form induces a nowhere vanishing holomorphic form on the quotient space $L^{*} / \bar{\Gamma}=X(\bar{\Gamma} ; L)^{*}$, i.e. $(X(\bar{\Gamma} ; L), o)$ is Gorenstein. Now, suppose that $m=-2$, i.e. $L^{2} \simeq T_{P}^{-1}$. The group $\mu_{2}$ acts naturally on $L$ as the subgroup $\{ \pm 1\}$ of $\mathbb{C}^{*}$ and the quotient $L / \mu_{2}$ is identified with $L^{2}$. Since this action commutes with the action of $\bar{\Gamma}$, we have a commutative diagram of unramified coverings:

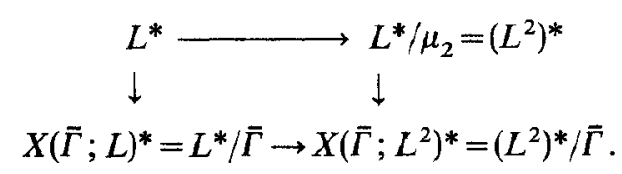

We already know that $\left(X\left(\bar{\Gamma} ; L^{2}\right), o\right)$ is Gorenstein. Thus, there exists a nowhere vanishing holomorphic 2 -form on $X\left(\bar{\Gamma} ; L^{2}\right)^{*}$. Its pull-back under the lower 
horizontal map in the diagram above is a nowhere vanishing holomorphic 2-form on $X(\bar{\Gamma} ; L)^{*}$. Hence $(X(\bar{\Gamma} ; L), o)$ is a Gorenstein singularity also. Conversely, if $(X(\bar{\Gamma} ; L), o)$ is a Gorenstein singularity, then the pull-back of the corresponding 2 -form to the cover $L^{*}$ is a nowhere vanishing holomorphic 2 -form on $L^{*}$. Thus, the singularity obtained by blowing down the zero section of $L$ into a normal point is a Gorenstein singularity. By Lemma 2 we obtain that $L^{m} \simeq T_{P}$ for some integer $m$ (as $\bar{\Gamma}$-bundles).

Let $P=H$. Suppose that $L^{m} \simeq T_{P}$ as $\bar{\Gamma}$-bundles for some integer $m$. Let $\left(L^{\prime}, G\right)$ be a standard reduction of $(L, \bar{\Gamma})$. Then $L^{\prime m} \simeq T_{C^{\prime}}$ as $G$-bundles. Since $T_{C^{\prime}}$ is a negative line bundle, $m$ must be positive. Assume that $m=1$. Choosing local coordinates $\left(z, t_{z}\right), t_{z}=d z$, we easily check that the 2 -form $t_{z}^{-2} d z \wedge d t_{z}$ is defined globally on $L^{*}$, does not vanish anywhere on $L^{\prime *}$ and is $G$-invariant. The rest of the proof in this case repeats word by word the proof in the previous case (with the final remark that $L^{m} \simeq T_{C^{\prime}}$ implies $L^{m} \simeq T_{P}$ ).

Let $P=\mathscr{C}$. If $\bar{\Gamma}$ is contained in the translation subgroup of $\operatorname{Aut}(P)$, then $C=P / \bar{\Gamma}$ is an elliptic curve and $L^{\prime}=L / \bar{\Gamma}$ is a negative line bundle on $C$. Since $T_{C}$ is trivial, by Lemma 1 the singularity $(X(\bar{\Gamma} ; L), o)$ is Gorenstein. If $\bar{\Gamma}$ contains an automorphism of finite order, then we consider a standard reduction $\left(C^{\prime}, L^{\prime}\right)$ of $(L, \bar{\Gamma})$. The singularity $(X(\bar{\Gamma} ; L), o)$ is obtained by dividing the singularity of the above type by a nontrivial action of a finite group. It is well-known that the obtained singularity is a rational singularity. Since its local fundamental group $\pi_{1}\left(X(\bar{\Gamma} ; L)^{*}\right)$ is infinite, it cannot be a Gorenstein singularity. Thus, $\bar{\Gamma}$ must be contained in the translation subgroup of $\operatorname{Aut}(P)$.

Remarks. 1. If $(X, o)$ is a hypersurface singularity given by a quasihomogeneous equation $f\left(z_{1}, z_{2}, z_{3}\right)=0$ of degree $d$ and weights $q_{1}, q_{2}, q_{3}$, then $(X, o) \simeq(X(\bar{\Gamma} ; L), o)$, where $L^{m} \simeq T_{P}$ and

$$
m=d-q_{1}-q_{2}-q_{3} .
$$

2. It is known that for a fixed link space $M$ the set of quasihomogeneous surface singularities $(X, o)$ with this link space is parametrized by a complex torus of $\operatorname{dim} g$, the Jacobian variety of the curve $C=X^{*} / \mathbb{C}^{*}[8]$. Proposition 1 implies that the set of Gorenstein singularities with the link space $M$, if non-empty, corresponds to the subgroup $(\mathbb{Z} / m \mathbb{Z})^{2 g}$ of this torus.

Recall the definition of the Seifert invariants of an automorphy factor $L$ [14]. This is a vector $\left(\operatorname{vdeg}(L) ; e_{1}, q_{1}, \ldots, e_{r}, q_{r}\right)$ defined as follows. Let $\left(L^{\prime}, G\right)$ be a standard reduction of $L$. We define $\operatorname{vdeg}(L)=\operatorname{deg}\left(L^{\prime}\right) / \# G$ (the virtual degree of $L$ ). This number is independent of a choice of the standard reduction $\left(L^{\prime}, G\right)$. Let $x_{1}, \ldots, x_{r}$ be representatives of the orbits of $\bar{\Gamma}$ in $P$ with non-trivial isotopy subgroups. We define $e_{i}$ as the order $\# \bar{\Gamma}_{x_{i}}$ of the corresponding isotopy subgroup $\bar{\Gamma}_{x_{i}}$. The group $\bar{\Gamma}_{x_{i}}$ acts on the tangent space $T(L)_{x_{i}}$ of the total space of $L$ at the point $x_{i}$ belonging to the zero section of $L$. The two eigensubspaces of $\bar{\Gamma}_{x_{i}}$ correspond to the tangent space $T(P)_{x_{i}}$ and the fibre $L_{x_{i}}$. Let $\left(\zeta, \zeta^{q_{2}}\right)$ be the corresponding eigenvalues (taken in the same order), where $\zeta$ is a primitive $e_{i}^{\text {th }}$ root of unity and $0 \leqq q_{i}<e_{i}$. This defines our numbers $q_{i}$. If $L$ is a non-ramified automorphy factor, then it is easy to see that $q_{i}$ is coprime to $e_{i}$. Also, it is known 
that the Seifert invariants of the automorphy factor $T_{P}$ are

$$
\left(2-2 g-r+e_{1}^{-1}+\ldots+e_{r}^{-1} ; e_{1}, 1, \ldots, e_{r}, 1\right),
$$

where $g$ is the genus of the curve $C=P / \bar{\Gamma}$.

The Seifert invariants of a negative non-ramified automorphy factor $L$ can be computed via a resolution of the singularity $(X(\bar{\Gamma} ; L), o)$ by using [9]. We refer to Theorem (2.3.1) of [14] for the statement of the corresponding result.

Corollary-Definition. A negative non-ramified automorphy factor $L$ on $P$ with respect to a discrete subgroup $\bar{\Gamma}$ of $\operatorname{Aut}(P)$ is called a Gorenstein automorphy factor if one of the following equivalent properties is satisfied:

(i) $(X(\vec{\Gamma} ; L), o)$ is a Gorenstein singularity;

(ii) $L^{m} \simeq T_{P}$ as automorphy factors for some integer $m$ and $\bar{\Gamma}$ is contained in the translation subgroup of Aut $(P)$ if $P=\mathbb{C}$. Moreover, (i) or (ii) implies

(iii) if $\left(v \operatorname{veg}(L) ; e_{1}, q_{1}, \ldots, e_{r}, e_{r}\right)$ are the Seifert invariants of $L$, then there exists an integer $m$ such that

$$
\begin{gathered}
m(\operatorname{vdeg}(L))=2-2 g-r+e_{1}^{-1}+\ldots+e_{r}^{-1}, \\
m q_{i}=1 \bmod e_{i}, \quad i=1, \ldots, r,
\end{gathered}
$$

where $g$ is the genus of the curve $P / \bar{\Gamma}$. If $g=0$, then (iii) implies (i) or (ii).

To see this we apply Proposition 1 and use the fact that two automorphy factors with the same Seifert invariants differ by the pull-back of a line bundle of degree 0 on $P / \bar{\Gamma}$.

\section{From Automorphy Factors to Lie Groups}

In this section we will prove that the "circle subbundle" of a Gorenstein automorphy factor is equivariantly diffeomorphic to a 3-dimensional Lie group. We deal separately with the each of the three planes $P$.

$P=\mathbb{P}^{1}(\mathbb{C})$

In this case $\left(T_{P}^{-1}\right)^{*}$ is isomorphic to the quotient of $\mathbb{C}^{2}-\{0\}$ by the involution $i:\left(z_{1}, z_{2}\right) \rightarrow\left(-z_{1},-z_{2}\right)$. We can choose the coordinates $\left(z_{1}, z_{2}\right)$ in $\mathbb{C}^{2}$ in such way that the group $\bar{\Gamma}$ Aut $(P)=$ PGL(1) lifts to a finite subgroup $\bar{\Gamma}$ of the subgroup $\operatorname{SU}(2)$ of $\operatorname{SL}(2, \mathbb{C})$ which preserves the Hermitean form $z_{1} \bar{z}_{1}^{\prime}+z_{2} \bar{z}_{2}^{\prime}$. The group SU (2) acts on the 3-sphere $S: z_{1} \bar{z}_{1}+z_{2} \bar{z}_{2}=1$ simply transitively by the restriction of its natural action in $\mathbb{C}^{2}$. Thus, we can identify $S^{3}$ with $S U(2)$ and the quotient $S^{3} / i$ with PSU(2). The projection $\mathbb{C}^{2}-\{0\} / i \rightarrow P$ induces an embedding of PSU(2) into $T_{P}^{-1}$. Its image is the unit circle subbundle $S$ of $T_{P}^{-1}$ with respect to the metric on $\left(T_{P}^{-1}\right)^{*}$ induced by the above Hermitean form on $\mathbb{C}^{2}$. It is clear that the diffeomorphism PSU(2) $\rightarrow S$ is $\bar{\Gamma}$-equivariant, where $\bar{\Gamma}$ acts on PSU(2) by left multiplication. Identifying $S$ with the quotient $\left(T_{\boldsymbol{P}}^{-1}\right)^{*} / \mathbb{R}_{+}$, we get a commutative 
diagram of differentiable maps:

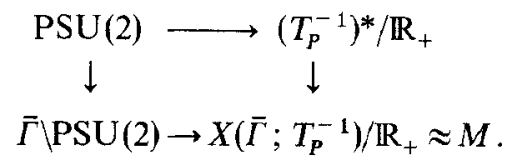

Here the horizontal maps are diffeomorphisms and the vertical maps are local diffeomorphisms.

The universal cover of PSU(2) is the double cover SU(2) $\rightarrow \mathrm{PSU}(2)$. This shows that

$$
M \underset{\text { diff }}{\approx} \bar{\Gamma} \backslash \mathrm{PSU}(2) \underset{\text { diff }}{\approx} \Gamma \backslash \mathrm{SU}(2) .
$$

If $L^{2} \simeq T_{P}^{-1}$, then $L^{*}$ is isomorphic to $\mathbb{C}^{2}-\{0\}$ and $\mathrm{SU}(2)$ is embedded into $L^{*}$ with the image $S^{3}$. The group $\bar{\Gamma}$ lifts isomorphically to a subgroup $\Gamma$ of $\operatorname{SU}(2)$ (necessary cyclic) and the diffeomorphism $\mathrm{SU}(2) \rightarrow S^{3}$ is $\Gamma$-equivariant. As in the case $L=T_{P}^{-1}$ we have a commutative diagram of differential maps:

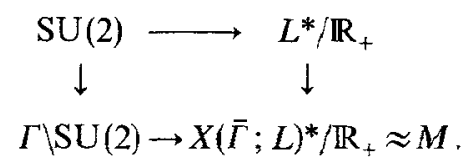

$P=H$

Let $L=T_{P}$. The tangent bundle $T_{P}$ has a riemannian PSL $(2, \mathbb{R})$-invariant metric $\operatorname{Im}(z)^{-1} d z$. The unit circle subbundle $S$ of $T_{\mathbf{P}}$ can be identified with the subbundle of the trivial bundle $P \times \mathbb{C}$

$$
S=\{(z, t) \in P \times \mathbb{C}: t=\operatorname{Im}(z)\} .
$$

The group $\operatorname{PSL}(2, \mathbb{R})=\operatorname{Aut}(P)$ acts on $T_{P}$ by the formula:

$$
g(z, t)=(g(z), \dot{g}(z) t), \quad g \in \operatorname{PSL}(2, \mathbb{R}) .
$$

Since $|\dot{g}(z)|=|c z+d|^{-2}$, where $g: z \rightarrow(a z+b)(c z+d)^{-1}$, one easily checks that $S$ is preserved under the action of $\operatorname{PSL}(2, \mathbb{R})$ on $T_{\mathrm{P}}$. Also one verifies that the action of $\operatorname{PSL}(2, \mathbb{R})$ on $S$ obtained in this way is simply transitive and defines a $\bar{\Gamma}$-equivariant diffeomorphism $\operatorname{PSL}(2, \mathbb{R}) \rightarrow S$, where $\bar{\Gamma}$ acts on $\operatorname{PSL}(2, \mathbb{R})$ by left multiplication.

Similarly to the previous case, we have a commutative diagram of differentiable maps

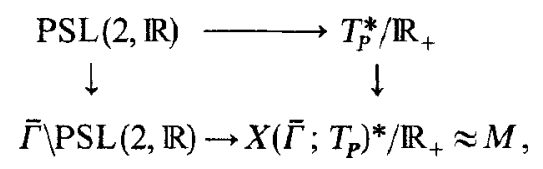

where the horizontal maps are diffeomorphisms and the vertical maps are local diffeomorphisms. 
The universal cover of $\operatorname{PSL}(2, \mathbb{R})$ is a simply connected Lie group $\operatorname{PSL}(2, \mathbb{R})^{\sim}$ which fits into a central extension

$$
1 \rightarrow \mathbb{Z} \rightarrow \operatorname{PSL}(2, \mathbb{R})^{\sim} \rightarrow \operatorname{PSL}(2, \mathbb{R}) \rightarrow 1 .
$$

Let $\Gamma$ be the subgroup of $\operatorname{PSL}(2, \mathbb{R})^{\sim}$ obtained by the restriction of this extension to the subgroup $\bar{\Gamma}$ of $\operatorname{PSL}(2, \mathbb{R})$. It fits into a central extension

$$
1 \rightarrow \mathbb{Z} \rightarrow \Gamma \rightarrow \bar{\Gamma} \rightarrow 1 \text {. }
$$

Clearly, we have

$$
M \underset{\text { diff }}{\approx} \bar{\Gamma} \operatorname{PSL}(2, \mathbb{R}) \underset{\text { diff }}{\approx} \Gamma \backslash \operatorname{PSL}(2, \mathbb{R}) \sim
$$

Now, if $L^{m} \simeq T_{\boldsymbol{P}}$, we consider as in Sect. 2 the unramified $\bar{\Gamma}$-equivariant cover

$$
L^{*} \rightarrow L^{*} / \mu_{m} \simeq T_{\mathbf{P}} \text {. }
$$

The inverse image $\bar{S}$ of the unit circle subbundle $S$ of $T_{P}$ is a unramified $m$-cover of $S$. It has a natural structure of a 3-dimensional Lie group $\bar{G}$ such that the projection $\bar{S} \rightarrow S$ is a homomorphism of Lie groups. The group $\bar{\Gamma}$ is a discrete subgroup of $\bar{G}$ and we have a $\bar{\Gamma}$-equivariant diffeomorphism $\bar{G} \rightarrow L^{*} / \mathbb{R}_{+}$. It induces a diffeomorphism

$$
\bar{G} \rightarrow X(\bar{\Gamma} ; L)^{*} / \mathbb{R}_{+} \approx M .
$$

Obviously, the universal cover of $\bar{G}$ is $\operatorname{PSL}(2, \mathbb{R})^{\sim}$ and the group $\bar{\Gamma}$ extends to a subgroup $\Gamma$ of $\operatorname{PSL}(2, \mathbb{R})^{\sim}$ such that

$$
\Gamma \backslash \operatorname{PSL}(2, \mathbb{R})^{\sim} \underset{\text { diff }}{\approx} \bar{\Gamma} \backslash \bar{G} \underset{\text { diff }}{\approx} M .
$$

$P=\mathbb{C}$

In this case $\bar{\Gamma}$ can be identified with a sublattice $\mathbb{Z} \cdot 1+\mathbb{Z} \tau$ of the additive group $\mathbb{C}$, where $\operatorname{Im}(\tau)>0$. The automorphy factor $L$ is identified with the trivial line bundle $P \times \mathbb{C}$ on which $\vec{\Gamma}$ acts by the formula:

$$
w:(z, t) \rightarrow\left(z+w, e_{w}(z) t\right), \quad w \in \vec{\Gamma} .
$$

Here, $e_{w}(z)$ is a theta factor chosen in the form

$$
e_{w}(z)=\exp \left(\pi H(z, w)+\frac{\pi}{2} H(w, w)\right) a(w),
$$

where $H\left(z, z^{\prime}\right)$ is a positive definite Hermitean form on $\mathbb{C}$ such that $\operatorname{Im}\left(H\left(w, w^{\prime}\right)\right) \in \mathbb{Z}$ for $w, w^{\prime} \in \bar{\Gamma}$ and $a: \bar{\Gamma} \rightarrow \mathbf{U}(1)$ is a map satisfying

$$
a\left(w+w^{\prime}\right)=a(w) a\left(w^{\prime}\right) \exp \left(\pi i \operatorname{Im}\left(H\left(w, w^{\prime}\right)\right)\right.
$$

(see, for example, [5]). In our one-dimensional case, we easily find that

$$
H\left(z, z^{\prime}\right)=k \operatorname{Im}(\tau)^{-1} z \bar{z}^{\prime}, \quad a(m+n \tau)=\exp (\pi i m n) a_{0}(m+n \tau),
$$

where $k$ is a positive integer and $a_{0}: \bar{\Gamma} \rightarrow \mathbf{U}(1)$ is a homomorphism of groups.

Let $S$ be the unit circle subbundle of $L$ given by

$$
S=\{(z, t) \in P \times \mathbb{C}:|t|=1\} .
$$


The projection $L^{*} \rightarrow L^{*} / \mathbb{R}_{+}$can be identified with the map

$$
L^{*} \rightarrow S, \quad(z, t) \rightarrow\left(z,|t|^{-1} t\right) .
$$

It is immediately seen that this map is $\bar{\Gamma}$-equivariant, where $\bar{\Gamma}$ acts on $S$ by the formula

$$
w:(z, t) \rightarrow(z+w, \exp (\pi i \operatorname{Im}(H(z, w)) a(w) t) .
$$

We make a Lie group from $S$ by defining

$$
(z, t) \cdot\left(z^{\prime}, t^{\prime}\right)=\left(z+z^{\prime}, t t^{\prime} \exp \left(\pi i \operatorname{Im}\left(H\left(z, z^{\prime}\right)\right) .\right.\right.
$$

It is immediately checked that the map $w \rightarrow(w, a(w))$ is a homomorphism of groups $\bar{\Gamma} \rightarrow S$. We identify $\bar{\Gamma}$ with its image under this map. Similarly to the two previous cases, we obtain a commutative diagram of differentiable maps

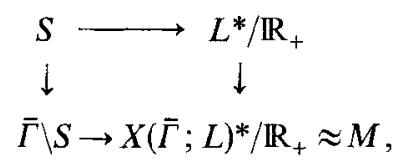

where the horizontal maps are diffeomorphisms and the vertical maps are local diffeomorphisms.

The universal cover of group $S$ is a simply connected Lie group $G^{\prime}$ whose elements are pairs $(z, c) \in \mathbb{C} \times \mathbb{R}$ multiplied by the rule:

$$
(z, c) \cdot\left(z^{\prime}, c^{\prime}\right)=\left(z+z^{\prime}, c+c^{\prime}+\frac{1}{2} \operatorname{Im}\left(H\left(z, z^{\prime}\right)\right) .\right.
$$

The projection $G^{\prime} \rightarrow S$ is a homomorphism of groups given by the formula:

$$
\exp :(z, c) \rightarrow(z, \exp (2 \pi i c)) \text {. }
$$

The group $\bar{\Gamma}$ extends to a discrete subgroup $\Gamma^{\prime}$ of $G^{\prime}$ consisting of the elements $(w, \tilde{a}(w)+\mathbb{Z})$, where $\tilde{a}: \bar{\Gamma} \rightarrow \mathbb{R}$ is a lifting of $a: \bar{\Gamma} \rightarrow \mathbf{U}(1)$ satisfying

$$
\tilde{a}\left(w+w^{\prime}\right)=\tilde{a}(w)+\tilde{a}\left(w^{\prime}\right)+\frac{1}{2} \operatorname{Im}\left(H\left(w, w^{\prime}\right)\right) \bmod \mathbb{Z} .
$$

If $w=m+n \tau$, we can define $\tilde{a}$ by

$$
\tilde{a}(w)=\frac{k}{2} m n .
$$

It is immediately checked that the left multiplication action of $\bar{\Gamma}$ on $S$ extends to the left multiplication action of $\Gamma^{\prime}$ on $G^{\prime}$ and

$$
\bar{\Gamma} \backslash G^{\prime} \underset{\text { diff }}{\approx} \bar{\Gamma} \backslash S \underset{\text { diff }}{\approx} M .
$$

To identify the pair $\left(G^{\prime}, \Gamma^{\prime}\right)$ with the pair $(G, \Gamma)$ of Type III) from Introduction, we define a representation $f: G^{\prime} \rightarrow \mathrm{GL}(3, \mathbb{R})$ :

$$
f(z, c)=\left[k a, k b, k c+\frac{k^{2}}{2} a b_{0}\right], \text { where } z=a+b \tau, \quad a, b \in \mathbb{R} .
$$


Since

$$
(a+b \tau, c) \cdot\left(a^{\prime}+b^{\prime} \tau, c^{\prime}\right)=\left(a+a^{\prime}+b \tau+b^{\prime} \tau, c+c^{\prime}+\frac{k}{2}\left(a b^{\prime}-a^{\prime} b\right)\right)
$$

and

$$
[a, b, c] \cdot\left[a^{\prime}, b^{\prime}, c^{\prime}\right]=\left[a+a^{\prime}, b+b^{\prime}, c+c^{\prime}+a b^{\prime}\right],
$$

we verify that $f$ is a homomorphism, its image is equal to the Heisenberg group $G$, and the image of $\Gamma^{\prime}$ is equal to the subgroup $\Gamma$ of $G$.

Summarizing, we get the first half of the theorem stated in Introduction:

Theorem 1. Let $M$ be the link space of a normal Gorenstein quasihomogeneous surface singularity. Then $M$ is diffeomorphic to a coset space $\Gamma \backslash G$, where $(G, \Gamma)$ is an admissible pair of groups.

\section{From Lie Groups to Automorphy Factors}

Here we prove the second part of the theorem from Introduction. The following result is well-known (see, for example, [12]):

Lemma 3. Let $G$ be a simply connected Lie group of dimension 3. Then $G$ is isomorphic to one of the following groups:

1) $G=\operatorname{SU}(2)$;

2) $G=\operatorname{PSL}(2, \mathbb{R})^{\sim}$;

3) $G$ is the Heisenberg group (a nilpotent group);

4) $G$ is an extension $1 \rightarrow \mathbb{R}^{2} \rightarrow G \rightarrow \mathbb{R} \rightarrow 1$ with $[G, G]=\mathbb{R}^{2}$;

5) $G=\mathbb{R} \times G^{\prime}$, where $G^{\prime}$ is the group of matrices $\left(\begin{array}{cc}a & b \\ 0 & 1 / a\end{array}\right), a \in \mathbb{R}_{+}, b \in \mathbb{R}$;

6) $G$ is the abelian group $\mathbb{R}^{3}$.

Proposition 2. Let $M$ be the link space of a quasihomogeneous normal surface singularity. Suppose that $M$ is diffeomorphic to a coset space $\Gamma \backslash G$, where $\Gamma$ is a discrete cocompact subgroup of a simply connected Lie group of dimension 3 . Then $G$ is one of the three Types 1)-3) from Lemma 3.

Proof. We may assume that $M$ is the link space of a singularity $X(\bar{\Gamma} ; L)$ corresponding to an automorphy factor $L$ on one of the three standard planes $P$. The group $\Gamma=\pi_{1}(M)$ is an extension of $\bar{\Gamma}$ with help of a cyclic group $K$. If $P=\mathbb{P}^{1}(\mathbb{C})$, then $\bar{\Gamma}$ is finite and $K$ is finite. The only Lie group $G$ from the list of Lemma 3 which contains a finite cocompact discrete subgroup is the group $\mathrm{SU}(2)$. If $P=H$, then as well-known, $\bar{\Gamma}$ is not solvable. This eliminates Cases 3) 6 ). Since $\bar{\Gamma}$ is infinite, we must be in Case 2). Finally, if $P=\mathbb{C}$, then $\bar{\Gamma}$ is a semi-direct product of a finite group and a lattice $\mathbb{Z}^{2}$. A quick glance at the list of Lemma 3 reveals that the finite part of $\bar{\Gamma}$ must be trivial and $G$ is not of Types 1) or 2). The singularity $X(\bar{\Gamma} ; L)$ in this case is a simple elliptic singularity: it is obtained by blowing down the zero section of a negative line bundle over an elliptic curve. The link $M$ is a circle bundle (non-trivial) over a torus. This easily implies that the extension 
$1 \rightarrow K \rightarrow \Gamma \rightarrow \bar{\Gamma} \rightarrow 1$ is non-trivial, and $\Gamma /[\Gamma, \Gamma] \simeq \mathbb{Z} \oplus \mathbb{Z} \oplus \mathbb{Z} / k \mathbb{Z}$, where $k=-c_{1}(L)$. This immediately eliminates Cases 5) and 6). Since $[G, G]=\mathbb{R}^{2}$ in Case 4), this case is also impossible.

Now, we can prove the second part of the theorem from Introduction.

Theorem 2. Let $M$ be the link space of a normal quasihomogeneous surface singularity. Assume that $M$ is diffeomorphic to a coset space $\Gamma \backslash G$, where $G$ is a simply connected 3-dimensional Lie group and $\Gamma$ its discrete subgroup. Then $M$ is diffeomorphic to the link space of a Gorenstein quasihomogeneous singularity.

Proof. By Proposition 2 the group $G$ must be of Types 1), 2) or 3) from Lemma 3. Let $Z$ be the center of $G$ and $p: G \rightarrow G / Z=\bar{G}$ be the projection. It is easy to see that $Z=\{ \pm 1\}, \bar{G}=\operatorname{PSU}(2) \subset \operatorname{Aut}\left(\mathbb{P}^{1}(\mathbb{C})\right)$ in Case 1$) ; Z=\mathbb{Z}, \bar{G}=\operatorname{PSL}(2, \mathbb{R})=\operatorname{Aut}(H)$ in Case 2$) ; Z=\mathbb{R}, \bar{G}=\mathbb{R}^{2}(\operatorname{Aut}(\mathbb{C})$ in Case 3). Also we see that $\bar{\Gamma}=\Gamma / \Gamma \cap Z$ is a discrete cocompact subgroup of $\bar{G}$. Let $S=G / \Gamma \cap Z$ and $q: S \rightarrow P$ be the composition of the map $p$ restricted to $S$ and the map $\bar{G} \rightarrow P$ corresponding to a choice of a point on $P$. It is easily checked that $q$ defines a structure of a $\bar{\Gamma}$-equivariant circle bundle over $P$. Let $L$ be the associated line bundle over $P$. Clearly, it is an automorphy factor with respect to $\bar{\Gamma}$. If $P \neq \mathbb{C}, Z / \bar{\Gamma} \cap Z$ is a finite cyclic group isomorphic to the group $\mu_{m}$ of $m^{\text {th }}$ roots of unity and $S / \mu_{m} \simeq \bar{G}$. As we saw in Sect. 2, we can identify $\bar{G}$ with the unit circle subbundle of $T_{P}^{-1}$ in case $P=\mathbb{P}^{1}(\mathbb{C})$ and of $T_{P}$ in case $P=H$. This implies that $L^{m}=L / \mu_{m}$ is $\bar{\Gamma}$-equivariant isomorphic to $T_{P}^{-1}$ if $P=\mathbb{P}^{1}(\mathbb{C})$ and to $T_{P}$ if $P=H$. By Proposition 1 , we obtain that $(X(\bar{\Gamma} ; L), o)$ is a Gorenstein singularity if $\boldsymbol{P} \neq \mathbb{C}$. Obviously, its link space is diffeomorphic to $M$ (both of them are diffeomorphic to the same coset space $\Gamma \backslash G$ ). It remains to consider the case, where $G$ is of Type 3), i.e. $P=\mathbb{C}$. In this case $M$ must be the link space of a simple elliptic singularity, necessary a Gorenstein singularity.

Remarks. 1 . Let $(X, o)$ be a cusp singularity, a normal surface singularity whose resolution graph is a cycle of nonsingular rational curves. It is well-known that all cusp singularities are Gorenstein singularities and their link space is diffeomorphic to a torus bundle over a circle. This easily implies that it is diffeomorphic to a coset space $\Gamma \backslash G$, where $G$ is a 3-dimensional Lie group of Type 4) of Lemma 3. The corresponding extension $1 \rightarrow \mathbb{R}^{2} \rightarrow G \rightarrow \mathbb{R} \rightarrow 1$ splits and $G$ can be described explicitly as $\mathbb{R}^{\mathbf{3}}$ with the group structure

$$
(a, b, c) \cdot\left(a^{\prime}, b^{\prime}, c^{\prime}\right)=\left(a+e^{c} a^{\prime}, b+e^{-c} b^{\prime}, c+c^{\prime}\right)
$$

(see [8]). Notice that there exists only one more group $G$ of Type 4) which admits a discrete cocompact subgroup. This is the universal covering of the Euclidean group, the group of orientation preserving isometries of $\mathbb{R}^{2}$ (see, for example, [12]). Using the classification of normal surface singularities whose local fundamental group is solvable [13], one can show that the cusp singularities are the only non-quasihomogeneous singularities whose link space is diffeomorphic to a coset space $\Gamma \backslash G,[8]$.

2. Using $[7,8,12]$ one can show that Proposition 2 has a true converse: A compact 3-dimensional homogeneous space $\Gamma \backslash G$ is diffeomorphic to the link space of a surface singularity if and only if the universal cover of $G$ is of Types 1)-4) of Lemma 3. Here in Case 4) $G$ must be the group $\mathbb{R}^{3}$ as above corresponding to cusp 
singularities. Also $G$ can be oriented so that the above is true for oriented manifolds; in general $\Gamma \backslash G$ with reversed orientation will neither be a homogeneous space [unless $G$ is of Type 4 or $G=\mathrm{SU}(2), \Gamma=\mathbb{Z} / 2 \mathbb{Z}$ ] nor a link space [unless $G$ is of Type 4) or $G=\mathrm{SU}(2), \Gamma$ is cyclic].

Acknowledgements. This work was done during my stays at the Mathematics Institute of the University of Warwick and the Max-Planck-Institut fuir Mathematik at Bonn. I am grateful to the both institutions for their hospitality and financial support.

\section{References}

1. Cartan, E. Sur la geometrie pseudo-conforme des hypersurfaces de deux variables complexes. Oeuvres II, 2, 1231-1304. Paris (1953)

2. Dolgachev, I.: Automorphic forms and quasihomogeneous singularities. Funct. Anal. Appl. 9, 67-68 (1975)

3. Dolgachev, I.: Algebraic properties of the algebras of automorphic forms. Proc. Conf, on Automorphic forms in honor of J. Lehner. Pittsburgh 1978 (to appear)

4. Milnör, J.: On the 3-dimensional Brieskorn manifolds. In: Knots, groups, and 3-manifolds. Ann. Math. Studies, Vol. 84, pp. 175-224. Princeton: Princeton University Press 1975

5. Mumford, D.: Abelian varieties. Oxford: Oxford University Press 1974

6. Neumann, W.: Brieskorn complete intersections and automorphic forms. Invent. Math. 42, 285-293 (1977)

7. Neumann, W.: A calculus for plumbing applied to the topology of complex surface singularities and degenerating complex curves. Trans. Am. Math. Soc. 268, 299-344 (1981)

8. Neumann, W.: Geometry of quasihomogeneous surface singularities. Proc. Symp. Pure Math. AMS 40, Vol. 2, 245-258 (1983)

9. Orlik, P., Wagreich, P.: Isolated singularities of algebraic surfaces with $\mathbb{C}^{*}$-action. Ann. Math. 93, 205-228 (1971)

10. Pinkham, H.: Normal surface singularities with $\mathbb{C}^{*}$-action. Math. Ann. 227, 183-193 (1977)

11. Poincaré, H.: Memoire sur les fonctions fuchsiennes. Acta Math. 1, 193-296 (1882)

12. Raymond, F., Vasquez, A.: 3-manifolds whose universal coverings are Lie groups. Topology Appl. $12,161-179$ (1981)

13. Wagreich, P.: Singularities of complex surfaces with solvable local fundamental group. Topology 11, 51-72 (1972)

14. Wagreich, P.: The structure of quasihomogeneous singularities. Proc. Symp. Pure Math. AMS 40, 593-624 (1983)

15. Yau, S.: Kohn-Rossi cohomology and its application to the complex Plateau problem. Ann. Math. 113, 67-110 (1981)

Received April 11, 1983; in revised form August 10, 1983 